\title{
Serial Tap Test of patients with idiopathic normal pressure hydrocephalus: impact on cognitive function and its meaning
}

\author{
Samanta Fabrício Blattes da Rocha ${ }^{1 *} \mathbb{0}$, Pedro André Kowacs ${ }^{1,2}$, Ricardo Krause Martinez de Souza', \\ Matheus Kahakura Franco Pedro', Ricardo Ramina' and Hélio A. Ghizoni Teive ${ }^{3}$
}

\begin{abstract}
Background: Idiopathic normal pressure hydrocephalus (INPH) is characterized by gait disturbance, urinary incontinence and cognitive decline. Symptoms are potentially reversible and treatment is based on cerebrospinal fluid shunting. The tap test (TT) is used to identify patients that will benefit from surgery. This procedure consists of the withdrawal of 20 to $50 \mathrm{~mL}$ of cerebrospinal fluid (CSF) through a lumbar puncture (LP) after which the symptoms of the triad are tested. Improvement in the quality and speed of gait are already recognized but cognitive improvement depends on several factors such as tests used, the time elapsed after LP for re-testing, and the number of punctures. Serial punctures may trigger similar conditions as external lumbar drainage (ELD) to the organism.
\end{abstract}

Objective: This study aimed to identify how serial punctures affect cognition to increase the sensitivity of the test and consequently the accuracy of surgical indication.

Methods: Sixty-one patients with INPH underwent baseline memory and executive tests repeatedly following the 2-Step Tap Test protocol (2-STT - two procedures of $30 \mathrm{~mL}$ lumbar CSF drainage separated by a 24-h interval). The baseline scores of INPH patients were compared with those of 55 healthy controls, and with intragroup post-puncture scores of the 2-STT.

Results: The group with INPH had lower performance than the control group in all cognitive tests (RAVLT, Stroop, CFT, FAR-COWA, FAB, MMSE, orientation, mental control), except for the forward digit span test ( $p=0.707)$. After conducting LP procedures, the Stroop test (words, colors and errors), RAVLT (stage A1, A6 and B1), and CFT (immediate and delayed R) scores were equal to those of the control group ( $p>0.05)$. The INPH group presented significant improvement after the first puncture in MMSE $(p=0.031)$ and in the Stroop Test (points) $(p<0.001)$. After the second puncture, subjects improved in orientation, MMSE, RAVLT (B1), Stroop (points, words, errors) and CFT (IR).

Conclusion: Progressive cognitive improvement occurred over the 2-STT and changes were more significant after the second LP in all cognitive domains except for RAVLT (A7). Encephalic alert system 'arousal' seems to participate in early improvements observed during 2-STT. The second LP increased the sensitivity of the drainage test to detect changes in cognitive variables, and consequently improved the quality of the method.

Keywords: Idiopathic normal pressure hydrocephalus, Tap test, Serial lumbar puncture, Cognition

*Correspondence: samanta@habilittare.com

${ }^{1}$ Neurological Institute of Curitiba (INC), Curitiba, Street Jeremias Maciel Perretto, 300, Curitiba, Paraná 81210-310, Brazil

Full list of author information is available at the end of the article

\section{Introduction}

Idiopathic normal pressure hydrocephalus (INPH) is characterized by the classic triad [1] of progressive symptoms: gait apraxia, dementia, and urinary incontinence

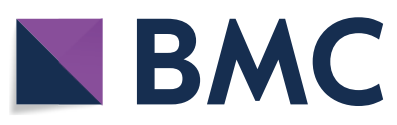

(c) The Author(s) 2021. This article is licensed under a Creative Commons Attribution 4.0 International License, which permits use, sharing, adaptation, distribution and reproduction in any medium or format, as long as you give appropriate credit to the original author(s) and the source, provide a link to the Creative Commons licence, and indicate if changes were made. The images or other third party material in this article are included in the article's Creative Commons licence, unless indicated otherwise in a credit line to the material. If material is not included in the article's Creative Commons licence and your intended use is not permitted by statutory regulation or exceeds the permitted use, you will need to obtain permission directly from the copyright holder. To view a copy of this licence, visit http://creativeco mmons.org/licenses/by/4.0/. The Creative Commons Public Domain Dedication waiver (http://creativecommons.org/publicdomain/ zero/1.0/) applies to the data made available in this article, unless otherwise stated in a credit line to the data. 
resulting from reasons that are not fully explained [2]. Cognitive alterations involve executive, attention, memory, and processing speed dysfunctions [3].

Symptoms of INPH can be alleviated with ventriculoperitoneal shunts [4]. To emulate this procedure, tests that promote the temporary removal of cerebrospinal fluid (CSF) are used to evaluate effects on gait and cognition. The most used methods are: a) the lumbar drainage test, known as Tap Test (TT); and b) the continuous lumbar drainage test (CLD). TT has high specificity (73-100\%) but low sensitivity (26-79\%) [5]. Marmarou et al. [5] and Ishikawa et al. [6] confirmed CLD to be more sensitive (50-100\%) than TT, but it is less popular due to invasiveness and morbidity $[7,8]$.

Aspects such as the amount of CSF drained and measurements of changes in gait and cognition may influence TT outcomes. There is evidence that the effects of CSF drainage in TT can extend beyond the time needed for CSF to regain its original volume and can last for $24 \mathrm{~h}$ or longer [9]. Despite the well-established methods to quantify gait improvement, there are still controversies about cognition improvement with TT [3].

Although memory mechanisms as learning, retention and retrieval are well known $[10,11]$, research on memory has not fully clarified the mechanisms of forgetting. The theory of interference [12-14] postulates that forgetfulness derives from the interference of one memory over another. There is evidence that the most important mechanisms are proactive interference (PI), in which prior learning affects later learning, and retroactive interference (RI), in which new information may interfere with prior learning. Thus, given the multiple mnemonic systems that are interacting mutually, studies using complex tests such as the Rey Auditory Verbal Learning Test (RAVLT) may help us to understand how this interaction occurs in a clinical condition that affects memory as INPH.

Ishikawa et al. [6] suggest that cognitive and urinary improvements may still occur up to one week after lumbar puncture. Serial lumbar punctures can produce, by analogy, similar physiological effects to CLD, with corresponding effects on gait and cognition. Thus, with multiple punctures, it may be possible that potential later cognitive amelioration can emerge earlier. The aim of present study was to evaluate the cognitive impact of serial tap test (STT) in patients with INPH.

\section{Material and methods}

The protocol was approved by the local regulatory committee for having followed the principles of the Helsinki Convention and its later amendments, as well as Brazilian guidelines disposed by resolution No. 466/2012, all individuals were adequately consented.

\section{Population}

\section{INPH population}

Study subjects were initially searched at the CSF Circulation Disturbance Research Program 2004-2017 files. This database contains information on well-established standardized procedures carried out at the Neurological Institute of Curitiba in suspected INPH subjects. Of the hundred and forty-eight subjects suspected of having INPH initially found, ten were not included for being aged 58 years or less, 27 for being classified as "unlikely", three for having been previously submitted to a neurosurgical procedure and further twelve for refusing to consent with the study procedures and other reasons (e.g.: missing data). Of the remaining 96 individuals with "probable" or "possible" INPH (according to the 2005 Euro-American Consensus) [5] were selected. Of those 96 subjects, 30 individuals were excluded due to comorbidities that could potentially impact cognitive functioning, such as active alcoholism, Parkinson's disease or parkinsonism, Alzheimer's disease, epilepsy, central nervous system [CNS] tumor, other neurological diseases such as vascular dementia, Lewy body disease, and multiple sclerosis; psychiatric disorders such as major depressive disorder, bipolar affective disorder, and attention deficit and cerebrovascular disorders (Fig. 1 and Table 1).

Individuals with a history of depression under control for more than three years or a history of mild TBI without permanent sequelae (cognitive or motor) were recruited (Table 2).

\section{Control population}

A control group was recruited from the community (patient's and or hospital staff's families and from an aged Baptist Church group). They were submitted to an interview about medical, scholarship and functional history, besides current health. Individuals suspected of neurological or psychiatric diseases were not included. An initial group of 57 individuals was selected. Of these, three subjects were excluded because of depression and brain trauma in the preceding 3 years.

\section{Study final population}

The remaining 66 INPH individuals and 54 control subjects underwent propensity score matching and both groups were homogenized according to age, gender, and educational level. This led to the exclusion of five individuals from the INPH group and 12 individuals from the control group. Data of 61 individuals from the INPH group and 42 from the control group were then compared (Fig. 2). 


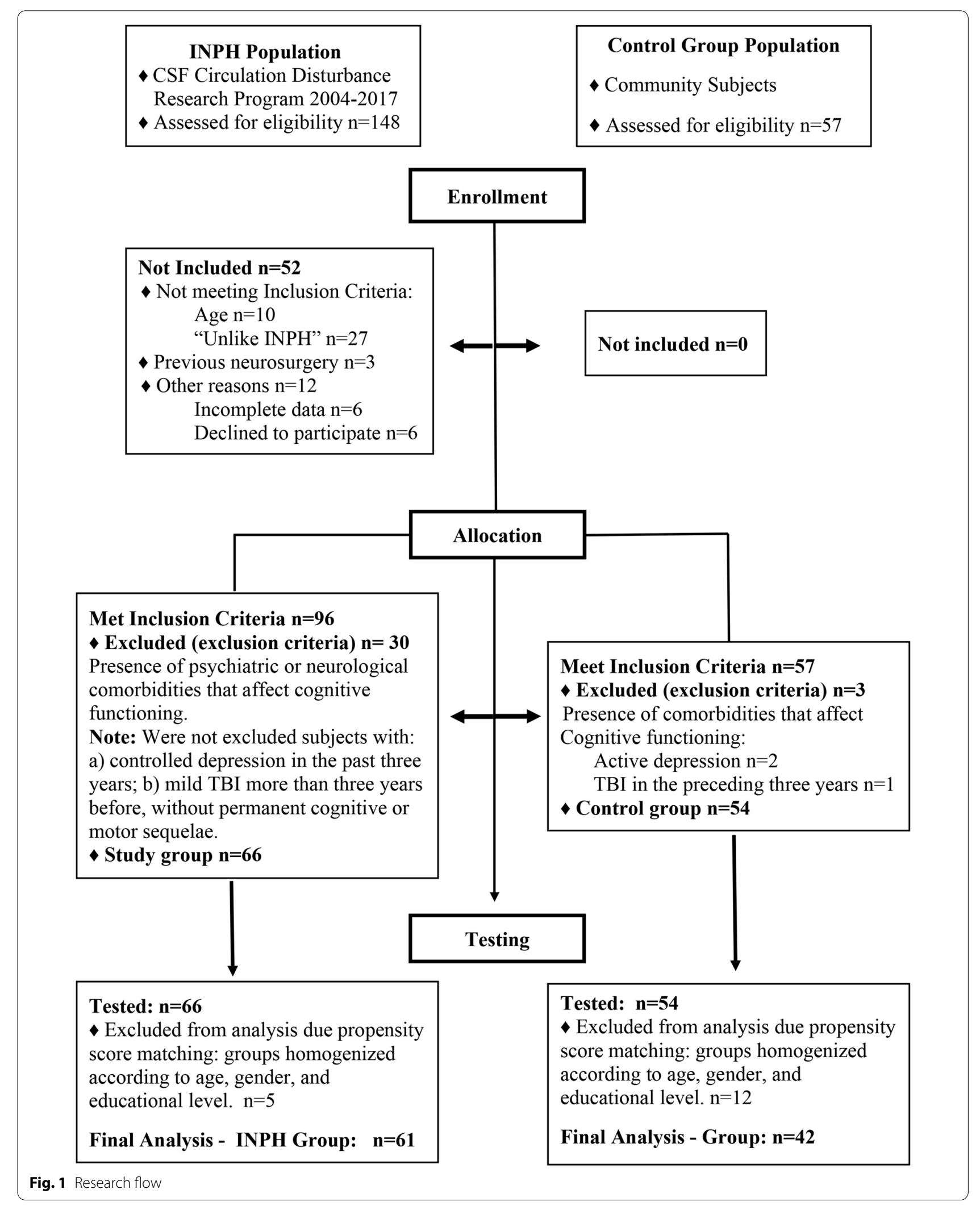


Table 1 Evolution over time of the classic HPN features in the INPH group

\begin{tabular}{llllrl}
\hline Variable & n & \% & M & SD & Range \\
\hline Age at onset of symptoms (years) & 61 & & 73.2 & 6.7 & \\
Time between start and STT (months) & 61 & & 27.7 & 26.2 & \\
Age at STT & 61 & & 75.5 & 6.5 & $58-86$ \\
Educational level_years & 61 & & 11.5 & 4.9 & $2-23$ \\
Male & 31 & & 50.8 & & \\
<One year with symptoms & 15 & 24.6 & & & \\
>One year with symptoms & 46 & 75.4 & & & \\
$\leq$ Two years with symptoms & 35 & 57.4 & & & \\
>Two years with symptoms & 26 & 42.6 & & & \\
Gait & 61 & 100 & & & \\
Cognition & 57 & 90 & & & \\
Sphincter & 45 & 69.8 & & & \\
One symptom (gait) & 4 & 6.7 & & & \\
Two symptoms (*) & 21 & 35 & & & \\
Complete triad & 35 & 58.3 & & \\
\hline
\end{tabular}

Values described in mean (M), standard deviation (SD) and percentages (\%). STT Serial Tap Test, $n$ number of individuals; $\leq$ : values equal to or less than the cutoff point; > : values higher than the cut-off point; ${ }^{*}$ gait apraxia associated with cognitive or sphincter symptoms

Table 2 Associated clinical conditions of the group with INPH

\begin{tabular}{|c|c|c|c|}
\hline Variable & Sample (N) & $\mathbf{n}$ & $\%$ \\
\hline Neurological events ${ }^{a}$ & & 16 & 26.22 \\
\hline $\mathrm{TBI}$ & & 8 & \\
\hline Stroke & & 6 & \\
\hline $\mathrm{SAH}$ & & 1 & \\
\hline $\mathrm{TIA}$ & & 1 & \\
\hline Hypertension & 57 & 36 & 63.2 \\
\hline History of Depression & 60 & 15 & 25 \\
\hline Diabetes & 61 & 15 & 24.6 \\
\hline Dyslipidemia & 61 & 11 & 18 \\
\hline Hypothyroidism & 60 & 7 & 11.7 \\
\hline Smoking & 60 & 3 & 5 \\
\hline
\end{tabular}

Data described in original sample number $(\mathrm{N})$, number $(\mathrm{n})$ and percentages (\%); $T B I$ traumatic brain injury, SAH subarachnoid hemorrhage, TIA transient ischemic attack. All individuals in the group with stroke $[n=6]$ presented a time interval between the stroke and STT greater than three years. Three subjects presented lower-impact ischemic stroke without sequelae. One individual had three episodes: in the first he became unable to wear his slippers, in the second there was loss of peripheral vision, and finally right hemiparesis in the third

\section{Procedures}

\section{Tap Test}

Patients were admitted to the hospital for two to three days. Two lumbar punctures were performed at intervals of at least $24 \mathrm{~h}$ apart (minimum $24 \mathrm{~h}$ and maximum $28 \mathrm{~h}$, to guarantee total LCR turnover) and $30 \mathrm{~mL}$ of CSF (M: $26 \pm 1.2$ ), was withdrawn in each, as described by Adams
[1]. CSF opening pressure was measured and a CSF sample was sent for laboratory analysis. The first LP was preceded by cognitive and gait examinations performed by a team of three neuropsychologists and four neurologists, respectively. After being examined for excluding postpuncture pain or headache [9] and between two and six hours after each LP, INPH subjects were tested again for both gait and cognitive examinations. Gait and cognition were never assessed within an interval shorter than one hour after the LP $(M=4,4 \pm 2,5)$. Neither persistent headache nor persistent pain after lumbar puncture were reported by individuals in this series, and further information regarding this issue can be found in a recent paper of our group [15].

To minimize interindividual bias, the neuropsychology team was constantly trained on study procedures. Furthermore, study data were discussed weekly and investigators remained blind to investigation subjects' diagnosis during study procedures. Gait protocol has been described elsewhere [16] and will not be discussed in this paper.

\section{Neuropsychological examination}

The neuropsychological assessment was performed during hospitalization. The average duration of the cognitive protocol was one hour and thirty minutes. Some data was not collected from patients with visual or motor impairment. The cognitive tests used were: Orientation (self-awareness, orientation in place, time and current context). Mental Control (usual and unusual activities: to speak the days of the week, the months of the year, to count (e.g.: 1-20) and after this, all the same activities are repeated in backward), FAR-COWA (The examinee must produce orally as many words as possible beginning with a specified letter during one minute (F-A-R) and thereafter he is asked to produce as many animal names as possible within a one-minute interval); Rey Auditory Verbal Learning Test (RAVLT): This is an episodic memory test: a 15-word list is read aloud (A list) for five consecutive trials (A1-A5). After trial A5, a distractor 15-word list is presented once and the examinee needs to learn it (B1); immediately after this, the examinee is asked to recall the A list (A6-immediate recall) and twenty minutes later again (A7-delayed recall); Rey Complex Figure Test (CFT): A visuospatial memory test: the examinee needs to learn a new complex figure by copying it. After a three-minute interval, he is asked to reproduce the figure as similar as possible without looking at it and thirty minutes later again); Digit Span Test (WMS-R): A two-step working memory test: 1 -the examinee is asked to repeat series of numbers in the same order that they are presented by the examiner; the quantity of digits increases progressively (digit span forward); 2-the examinee is 


\section{INPH group - 8-Step Serial Tap Test}

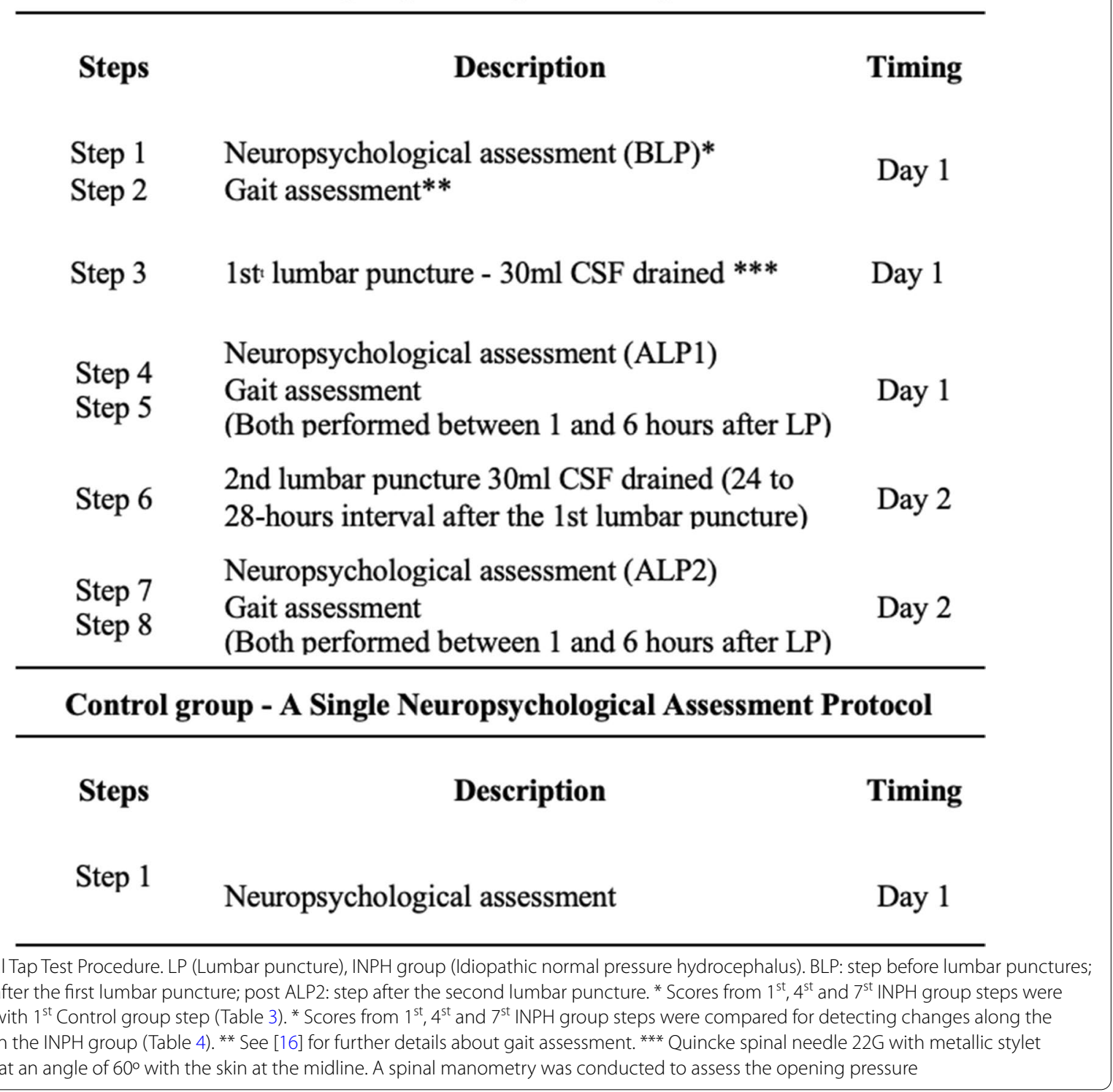

asked to repeat similar series but in backward (digit span backward). In our protocol both parts are scored separately; Stroop test (University of Victoria Version): This is an attention test: the examinee is presented to three cards. In the first card, there are 24 colored dots (blue, red, yellow, green). The subject is required to name the colors in which the stimuli are printed as fast as possible. In the second part, neutral words (when, where, what) are printed in the same colors of the dots and the subject needs to name the colors while ignoring the words. In the third part, the words blue, red, yellow and green are printed in conflicting colors (e.g.: the word "blue" printed in red color). Individuals are asked to name the colors printed again while avoid reading the words (name of colors). Scores are calculated based on the speed to perform the tasks and on the number of errors only in the third part; Mini Mental Status Examination (MMSE): This is a 30-point scoring test composed of "Introduction" section-items of orientation, memory and attention (21 points); "Materials" section-items of naming, follow verbal and written commands, writing a sentence and drawing a figure with intersections (9 points); Frontal Assessment Battery (FAB): This is a brief battery consisting of six subtests: similarities, lexical fluency, motor 
series, conflicting instructions, Go-No-Go test and prehension behavior). Each subtest can be scored from 0 to 3 points. The score is based on the sum of the six test scores.

To avoid a learning effect, some tests were applied in up to three different versions. The tests used in this study are among the most cited in INPH literature $[17,18]$.

\section{Matching procedure}

Patients with IHPH and control subjects were matched using propensity scores (PS). Three variables were selected to estimate PS: age, sex and formal education. The propensity score was calculated using logistic regression and patients with IHPH were matched with controls using the nearest neighbor technique with a predefined caliper of 0.2 and considering the ratio 1:1. This matching procedure was performed using the MatchIt package for R.16.

\section{Statistics}

A propensity score model was used to homogenize the INPH and the control group considering a logistic regression model and conditioning the variables of age, sex and formal education.

To analyze the effect of STT on cognitive test results of the INPH group in comparison with the control group, $\mathrm{Z}$ scores were calculated for each INPH patient regarding mean $(\mathrm{M})$ and standard deviation (SD) of the control group. The $\mathrm{Z}$ scores of variables whose improvements were equivalent to smaller values, such as run time number of errors, variables dots, words and colors, and errors of the Stroop test, were multiplied by -1 (inverted). Thus, in all variables, the highest values of the $\mathrm{Z}$ score corresponded to the participants' best results.

Results of quantitative variables were described by means and standard deviations. Categorical variables were presented in frequencies and percentages. Comparison between the INPH and control groups, concerning categorical variables, was conducted using Fisher's Exact Test. Comparison of either two disease-defined groups or clinical factors, relative to quantitative variables, was conducted using either Student's t-test for independent samples or the non-parametric Mann-Whitney test. More than two groups were compared considering the nonparametric Kruskal-Wallis test. Normality condition was evaluated through the Kolmogorov-Smirnov test. Values of $\mathrm{p}<0.05$ indicate statistical significance. Data were analyzed using the IBM Corp. Released 2011. IBM SPSS Statistics for Windows, Version 20.0. Armonk, NY: IBM Corp.

After propensity score matching, from the 66 subjects with INPH and the 54 control subjects initially recruited, $61 \mathrm{INPH}$ subjects and 42 control subjects remained for the statistical analysis. These groups were balanced regarding age $(\mathrm{p}=0.056)$, sex $(\mathrm{p}=0.231)$ and educational level $(\mathrm{p}=0.549)$.

Statistical analysis was supervised by a professional statistician.

\section{Results}

Regarding cognitive performance before the first LP, the INPH group scored lower than controls in every test except for the forward Digit Span Test. Values before and after the LP were also compared with control group scores (Table 3). The INPH group presented a similar score to the control group in the forward Digit Span Test in all steps of the serial drainage test. However, other measures of verbal memory, such as RAVLT-A1 and B1, improved after LP.

The INPH group scored lower than controls in FAB and MMSE scales before LP and after the two LP remained lower than those of the control group (Mann-Whitney, $\mathrm{p}=0.017$ and $\mathrm{p}=0.034$, respectively).

Table 4 shows the comparisons among the three steps of the cognitive test for all tests applied during STT.

Table 5 summarizes all variables with any significant improvement among the three testing steps, except for RAVLT-A7 (comparison between the moment before lumbar puncture and after the second lumbar puncture), which presented worsening.

An additional analysis was performed considering the previous history of associated clinical conditions of the INPH group, even if these conditions were not present for more than three years before the research period (Table 2). No differences were observed between subgroups with a history of previous depression, traumatic brain injury, or arterial hypertension. The stroke group presented better performance than the group without stroke in orientation (comparison between the moment before lumbar puncture and after first lumbar puncture) and the B1 memory RAVLT item (comparison between moment after first lumbar puncture and after second lumbar puncture; $\mathrm{p}=0.034$ and $\mathrm{p}=0.047$, respectively). All stroke individuals presented INPH symptoms for less than a year and did not report any cognitive permanent sequelae after the stroke event. Participants with diabetes presented greater mental slowness when compared with nondiabetic individuals $(\mathrm{p}=0.029)$, even after the second LP.

\section{Discussion}

INPH is one of the many diseases that can affect both motor and non-motor circuitry of the basal ganglia, and cause motor, autonomic, cognitive and behavioral symptoms [19]. Our study aimed to focus only on 
Table 3 INPH Pre-LP x post-LP1 and pre x post-LP2 cognitive scores, and control group cognitive scores

\begin{tabular}{|c|c|c|c|c|c|c|c|c|c|c|c|}
\hline \multirow[t]{2}{*}{ Variable } & \multirow[t]{2}{*}{$n$} & \multicolumn{3}{|l|}{ BLP } & \multicolumn{3}{|l|}{ ALP1 } & \multicolumn{2}{|l|}{ ALP2 } & \multicolumn{2}{|l|}{ CG } \\
\hline & & $M(S D)$ & $\mathbf{p}^{*}$ & $n$ & $M(S D)$ & $\mathrm{p}^{* *}$ & $\mathrm{n}$ & $M(S D)$ & $\mathrm{p}^{* * *}$ & $n$ & $M(S D)$ \\
\hline OR & 61 & $9.6(2.7)$ & $<0.001$ & 59 & $9.9(2.8)$ & $<0.001$ & 55 & 9.9 (2.9) & 0.001 & 42 & $11.7(0.8)$ \\
\hline$M C$ & 60 & $6.1(2.3)$ & 0.002 & 57 & $6.2(2.2)$ & 0.002 & 55 & $6.2(2.2)$ & 0.002 & 42 & $7.5(1.1)$ \\
\hline DS-For & 59 & $5.4(2.1)$ & 0.707 & 55 & $5.7(2.2)$ & 0.885 & 54 & $5.7(2.2)$ & 0.606 & 42 & $5.6(1.7)$ \\
\hline DS-Back & 59 & $3.3(1.8)$ & 0.003 & 55 & $3.4(1.9)$ & 0.026 & 54 & $3.4(1.9)$ & 0.015 & 39 & $4.2(1.3)$ \\
\hline FAR & 58 & $20.7(12.1)$ & $<0.001$ & 54 & $22.4(13.0)$ & 0.008 & 52 & $23.9(13.1)$ & 0.051 & 41 & $29.6(10.6)$ \\
\hline Animals & 58 & $9.3(4.9)$ & $<0.001$ & 53 & $9.2(4.7)$ & $<0.001$ & 53 & $9.6(5.3)$ & $<0.001$ & 41 & $13.9(4.1)$ \\
\hline $\mathrm{A} 1$ & 58 & $3.1(1.4)$ & 0.014 & 54 & $3.4(1.7)$ & 0.093 & 52 & $3.4(1.6)$ & 0.146 & 42 & $4.0(1.8)$ \\
\hline A5 & 57 & $6.0(3.1)$ & $<0.001$ & 54 & $6.6(2.9)$ & $<0.001$ & 52 & $6.0(2.8)$ & $<0.001$ & 42 & $9.6(2.5)$ \\
\hline Total & 57 & $24.5(10.4)$ & $<0.001$ & 54 & $26.6(10.5)$ & $<0.001$ & 52 & $25.3(10.6)$ & $<0.001$ & 42 & $37.0(10.3)$ \\
\hline B1 & 56 & $2.7(1.7)$ & 0.010 & 53 & $2.7(1.5)$ & 0.007 & 51 & $3.3(2.0)$ & 0.293 & 42 & $3.7(1.7)$ \\
\hline A6 & 59 & $2.7(1.7)$ & 0.010 & 55 & $2.7(1.5)$ & 0.007 & 55 & $3.3(2.0)$ & 0.293 & 42 & $3.7(1.7)$ \\
\hline A7 & 58 & $3.2(2.7)$ & $<0.001$ & 56 & $3.0(2.8)$ & $<0.001$ & 55 & $2.5(2.5)$ & $<0.001$ & 42 & $6.7(2.9)$ \\
\hline S-Dots ${ }^{\mathrm{a}}$ & 53 & $31.0(24.8)$ & $<0.001$ & 53 & $25.0(19.3)$ & 0.038 & 49 & $23.1(16.8)$ & 0.014 & 41 & $17.4(5.3)$ \\
\hline S-Word ${ }^{b}$ & 52 & $42.4(31.6)$ & $<0.001$ & 52 & $34.9(25.8)$ & 0.023 & 47 & $30.0(19.0)$ & 0.080 & 41 & $25.1(9.1)$ \\
\hline S-Colors ${ }^{c}$ & 52 & $58.6(32.4)$ & 0.003 & 49 & $52.8(42.7)$ & 0.255 & 47 & $46.1(26.4)$ & 0.479 & 39 & $42.8(18.9)$ \\
\hline S-Error ${ }^{d}$ & 46 & $8.1(8.8)$ & $<0.001$ & 46 & $6.2(7.1)$ & 0.002 & 39 & $4.7(6.0)$ & 0.092 & 41 & $2.3(2.9)$ \\
\hline CFT-cop & 34 & $24.0(9.4)$ & 0.016 & 33 & $22.5(9.5)$ & 0.002 & 29 & $24.2(9.4)$ & 0.047 & 42 & $28.7(7.1)$ \\
\hline CFT- IR & 34 & 7.5 (6.0) & 0.003 & 31 & $10.0(8.1)$ & 0.159 & 27 & $11.5(8.8)$ & 0.389 & 42 & $12.2(6.8)$ \\
\hline CFT- DR & 27 & $5.4(4.5)$ & $<0.001$ & 26 & $10.9(11.8)$ & 0.098 & 20 & $9.8(9.0)$ & 0.108 & 39 & $11.8(6.0)$ \\
\hline
\end{tabular}

All values described in mean (M) and standard deviation (SD); $n$ : number of individuals; BLP: step before lumbar punctures; ALP1: step after the first lumbar puncture; ALP2: step after the second lumbar puncture. Source tests: OR: orientation; MC: mental control; DS-FOR: forward digit span; DS-BACK: backwards digit span; FAR: verbal fluency test; RAVLT: Rey auditory verbal learning test; S-Dots ${ }^{\text {a }}$ : Stroop Test-dots step; S-Word ${ }^{\text {b }}$ : Stroop test - words step; S-Colors ${ }^{c}$ : Stroop test- colors step; S-Error ${ }^{d}$ : Stroop test - number of errors in the colors step; CFT-cop: Copy of Rey complex figure test; CFT-IR: Immediate reproduction of Rey complex figure test; CFT-DR: Delayed Reproduction of Rey complex figure test; MMSE Mini mental state examination, FAB frontal evaluation battery. P values: * non-parametric MannWhitney test, $\mathrm{p}<0.05$

cognitive-behavioral manifestations of INPH. The STT protocol was carried out at a hospital ward, avoiding the need for commuting, an aspect that contributed to its acceptance by patients and family. Hospitalization allowed all stages of the procedure to be controlled regarding external interferences on test results.

Serial drainage testing resulted in lower morbidity than continuous lumbar drainage $[8,20]$. Moreover, the second LP may increase the sensitivity of STT once detects more changes in cognitive variables, thus improving the method.

INPH subjects had lower performance than controls before LP in all tests regarding selective attention measures (words and colors in the Stroop test), distraction resistance (errors in the Stroop test), immediate and late visual memory (CFT). Similar results were described by Katzen et al. [21]. The exceptions to this finding in our study were the Digit Span Test. The fact that the Digit Span Scores were calculated forward and backward separately, probably triggered the differences regarding other studies [19, 22, 23], perhaps due to a ceiling effect in control individuals. However, other measures of supraspan, such as A1 and B1 of RAVLT, showed improvement.
The scores of the INPH group before LP were lower than the control group scores in both screening scales (MMSE and FAB) as previously observed by Katzen et al. [21] and Saito et al. [23]. Both scores remained lower after LPs, which reinforces the finding of severely compromised cognitive function in this population.

Eight cognitive items improved along with the STT, but the most paradoxical result was the decline of the RAVLT-A7 item, contrasting with the improvement seen in other tests. Serial Tap Test is an extensive protocol composed of several tasks applied to an elderly population. RAVLT-A7 item is the last phase carried out on the second day of examination and, for this reason, probably fatigue may have contributed to this result.

Significant improvement in $\mathrm{A}^{*}$ scores (Table 5) probably reflect an enhancement in alertness [24]. The complex relationships between alertness and attention have been adequately discussed in the literature [25].

It seems likely that both the enhancement in alertness and the decompression of frontostriatal circuits promote a gradual improvement in many cognitive aspects, especially in the dots and words variables of the Stroop test, in which subjects reacted faster after LP. Participants also showed improvement in their ability to inhibit impulsive 
Table 4 Comparison of the three assessment moments of the INPH group

\begin{tabular}{|c|c|c|c|c|c|c|}
\hline Original test & Variable & $n$ & $\begin{array}{l}\text { BLP } \\
\text { Mean } \pm \text { SD }\end{array}$ & ALP1 & $\begin{array}{l}\text { ALP2 } \\
\text { (Z Score) }\end{array}$ & $\mathrm{p}^{*}$ \\
\hline OR & Orientation & 54 & $-2.7 \pm 3.5$ & $-2.4 \pm 3.7$ & $-2.2 \pm 3.8$ & 0.018 \\
\hline$M C$ & Mental control & 53 & $-1.3 \pm 2.2$ & $-1.1 \pm 2.1$ & $-1.2 \pm 2.1$ & 0.156 \\
\hline \multirow[t]{2}{*}{ DS } & Forward & 51 & $-0.1 \pm 1.3$ & $0.1 \pm 1.3$ & $0.1 \pm 1.3$ & 0.327 \\
\hline & Backward & 51 & $-0.7 \pm 1.4$ & $-0.6 \pm 1.4$ & $-0.6 \pm 1.4$ & 0.600 \\
\hline \multirow[t]{2}{*}{ VF } & FAR & 28 & $-1.2 \pm 1.6$ & $-1 \pm 1.8$ & $-0.9 \pm 1.5$ & 0.053 \\
\hline & Animals & 49 & $-1.1 \pm 1.2$ & $-1.2 \pm 1.1$ & $-1 \pm 1.3$ & 0.753 \\
\hline \multirow[t]{6}{*}{ RAVLT } & A1 & 50 & $-0.5 \pm 0.8$ & $-0.5 \pm 0.8$ & $-0.4 \pm 0.9$ & 0.490 \\
\hline & A5 & 50 & $-1.5 \pm 1.2$ & $-1.2 \pm 1.1$ & $-1.5 \pm 1.1$ & 0.306 \\
\hline & Total A1-A5 & 50 & $-1.2 \pm 1$ & $-1.1 \pm 0.9$ & $-1.2 \pm 1$ & 0.113 \\
\hline & B1 & 49 & $-0.6 \pm 1$ & $-0.6 \pm 0.8$ & $-0.3 \pm 1.2$ & 0.024 \\
\hline & A6 & 51 & $-1.2 \pm 0.9$ & $-1.3 \pm 0.9$ & $-1.5 \pm 0.8$ & 0.081 \\
\hline & A7 & 51 & $-1.3 \pm 0.9$ & $-1.4 \pm 0.9$ & $-1.5 \pm 0.8$ & 0.048 \\
\hline \multirow[t]{4}{*}{ STROOP } & S-Dots & 45 & $-2.2 \pm 4.5$ & $-1.4 \pm 3.3$ & $-1.2 \pm 3.1$ & $<0.001$ \\
\hline & S-Words & 43 & $1.5 \pm 3.3$ & $1 \pm 2.5$ & $0.7 \pm 2.1$ & 0.031 \\
\hline & S-Colors & 42 & $-0.6 \pm 1.6$ & $-0.5 \pm 2$ & $-0.3 \pm 1.4$ & 0.541 \\
\hline & S-Errors & 36 & $-1.9 \pm 3.1$ & $-1.4 \pm 2.4$ & $-0.9 \pm 2.2$ & 0.010 \\
\hline \multirow[t]{3}{*}{ CFT } & CFT-Cop & 26 & $-0.6 \pm 1.3$ & $-0.8 \pm 1.4$ & $-0.8 \pm 1.3$ & 0.254 \\
\hline & CFT-IR & 24 & $-0.6 \pm 0.9$ & $-0.4 \pm 1.1$ & $0 \pm 1.3$ & 0.006 \\
\hline & CFT-DR & 15 & $-1 \pm 0.8$ & $0 \pm 2.3$ & $-0.4 \pm 1.4$ & 0.167 \\
\hline MMSE & MMSE & 48 & $-1.9 \pm 2.6$ & $-1.6 \pm 2.8$ & $-1.4 \pm 3$ & 0.021 \\
\hline$F A B$ & $F A B$ & 28 & $-1.2 \pm 1.6$ & $-1 \pm 1.8$ & $-0.9 \pm 1.5$ & 0.188 \\
\hline
\end{tabular}

All values converted and described in Z scores. SD standard deviation, $n$ number of individuals, BLP step before lumbar punctures, ALP1 step after the first lumbar puncture, post $A L P 2$ step after the second lumbar puncture. Source tests: $O R$ orientation, $M C$ mental control, DS digit span, VF verbal fluency test, FAR lexical fluency; Animals: semantic fluency; RAVLT: Rey auditory verbal learning test; S-Dots: Stroop Test-dots step; S-Word: Stroop test - words step; S-Colors: Stroop test- colors step; S-Error: Stroop test - number of errors in the colors step; CFT-cop: Copy of Rey complex figure test; CFT-IR: Immediate reproduction of Rey complex figure test; CFT-DR: Delayed Reproduction of Rey complex figure test; MMSE mini mental state examination, FAB frontal evaluation battery. P values: * Non-parametric Friedman test, $\mathrm{p}<0.05$

Table 5 Analysis of the differences of three evaluation moments of the INPH group

\begin{tabular}{|c|c|c|c|c|}
\hline \multirow[t]{3}{*}{ Cognitive variable } & \multicolumn{3}{|c|}{ Difference between steps } & \multirow[t]{3}{*}{$\mathrm{p}$} \\
\hline & A* scores & B* scores & $C^{*}$ scores & \\
\hline & pre-LP x post-LP1 & pre-LP x post-LP2 & post-LP1 x post-LP2 & \\
\hline OR_orientation & 0.121 & 0.004 & 0.177 & 0.018 \\
\hline MMSE & 0.031 & 0.008 & 0.614 & 0.021 \\
\hline RAVLT—B1 & 0.711 & 0.028 & 0.011 & 0.024 \\
\hline RAVLT—A7* & 0.153 & 0.014 & 0.290 & 0.048 \\
\hline ST—dots & $p<0.001$ & $p<0.001$ & 0.855 & $p<0.001$ \\
\hline ST-words & 0.069 & 0.010 & 0.422 & 0.031 \\
\hline ST-errors & 0.241 & 0.002 & 0.053 & 0.010 \\
\hline CFT- IR & 0.111 & 0.001 & 0.066 & 0.006 \\
\hline
\end{tabular}

P values: * Non-parametric Friedman test, $\mathrm{p}<0.05$; Post-hoc analysis with comparisons of groups. LP: lumbar puncture. Variable with its respective test of origin: OR: orientation; MMSE: Mini Mental State Examination; RAVLT-B1: B list of the Rey verbal learning test; RAVLT-A7: late recall [20 min after the list A6] of the Rey verbal learning test; ST-dots: first step [dots] of the Stroop test in the University of Victoria version; ST-words: second step [words] of the Stroop test in the University of Victoria version; ST-errors: numbers of errors in the third step [colors] of the Stroop test in the University of Vitoria version; CFT-IR: Immediate reproduction [after $3 \mathrm{~min}$ ] of the Rey complex figure test 
responses and to resist distractions (errors in the Stroop Test) [26]. Perhaps enhanced spatiotemporal perception revealed by the orientation test is also secondary to a better state of alertness. Isik et al. [27] performed serial punctures in the INPH patients group (mean duration interval of $7.4 \pm 5.7$ months between the first and second LP and a mean duration of $8.5 \pm 3.8$ months between second and third punctures). Each time, they tested these patients before LP and $24 \mathrm{~h}$ after. They found not a significant difference in the Stroop Test comparisons, at the first and second puncture moments. This result contrasts with what we found out. The first explanation is that our time interval between LP and the neuropsychological assessment was shorter (mean $4 \mathrm{~h}$ ). Although INPH physiopathology is not so far well explained, immediate and delayed mechanisms may be involved. A decompression effect may be immediately releasing attentional circuits, promoting a cognitive enhancement in the alertness status. This aspect, however, does not appear to be the only mechanism involved in INPH cognitive dysfunction.

Several other hypotheses were formulated to explain the complex pathophysiology of this disease, some of which involve cerebral parenchyma and CNS blood vessels, accumulation of toxic metabolites in CSF, and transependymal CSF permeation with cell and axonal damage $[15,19,28-32]$.

A similar effect on step A1 and B1 of RAVLT could be expected due to this gain in alertness since the structure of the two lists of words is similar. Despite the improvement seen in B1 $(p=0.024)$, the same was not found in A1 $(p=0.490)$. The structure of RAVLT may explain this difference since $\mathrm{B} 1$ is presented as a distraction element between the learning curve (A1-A5) and the immediate recall (A6) [33], whereas $\mathrm{A} 1$ is the first list presented in the test.

According to the theory of interference [12-14], forgetfulness can be understood as the interference of one memory over another. In RAVLT, a proactive interference (PI) is when prior learning affects later learning, but also a retroactive interference (RI) can occur, in which further learning affects the recovery of previously learned information. Thus, the improvement seen in B1 reflects a decrease in PI, but, in contrast, there was no change in RI $(\mathrm{A} 6-\mathrm{p}=0.081)$.

Time spent on the STT and the differences in the $\mathrm{A}^{*}$, $B^{*}$, and $C^{*}$ scores indicated cognitive evolution over the two days of examination. Time reported being required for cognitive improvement after LP varies among authors from 30-60 min to a week [8, 34-36]. However, all cognitive studies regarding TT screened for changes after a single puncture $[4,6,8,9,36-39]$. The present study is so far the first to systematically use serial lumbar punctures and systematically retest cognition over steps and time. Significant improvement in several cognitive domains in such a short time interval, compared with those reported in previous literature, suggests that the changes detected were a result of repeated punctures rather than merely the passage of time. Therefore, according to our results, mental speed, the earliest improved function after LP, continues improving after the second LP. It is likely that other skills may improve because of mental speed increasing, e.g., phonetic, and lexical verbal fluency tasks. These kinds of tests depend on language and executive functions. NPHI is not presumed to affect directly cortical functions as language fluency, but to mechanically compress the periventricular frontostriatal circuits and to cause transependymal CSF leakage and parenchymal edema, affecting language pathways speed. Thereafter, the progressive release of this circuitry may ameliorate its functioning.

Some limitations of this study should be considered. No retest in the control group was performed. Comparisons between the INPH group after LP and the control group after mental function re-testing would clearly define whether the changes observed in INPH subjects were not a learning effect (LE). LE may depend on individuals' characteristics as age and performance baseline [40]. A recent review [41] identified a lower LE in individuals with dementia as compared to normal aging individuals [42-45]. Our series of INPH individuals scored significantly below the normal aging group in all tests (except for the "digit span backward" subtest), a finding congruent with a cognitive decline. A single study about LE in INPH was published by Solana et al. [40] reported no learning effect in the INPH population when reapplying the same cognitive tests over four consecutive days. Our testing protocol started before Solana et al. [40] publication and has slight differences from their protocol, but the tests we have used are among the most used in INPH literature and there is a vast literature about patients' performance on them [17, 19, 21, 22, 35].

LE may depend on test characteristics as well, for example, a short-time-interval can result in a strong LE [40], and we sought for alternative testing to reduce the influence of this variable.

Lack of data on depression was also a gap in the study. However, self-evaluative scales have a limited effect on this population due to difficulties in differentiating depressive symptoms from frontal dysfunction. Kito et al. [46] observed that apathy is the most common neuropsychiatric disorder in this population and that this symptom has a high correlation with cognitive symptoms of the triad. Apathy is considered a symptom of somnolence-sopor-coma disorder (SSCD) [24, 47], as well as the emotional asthenic syndrome or apathetic-abulic 
syndrome [41]. The correlation between apathy and executive dysfunction has been attributed to the association of this symptom with INPH dysfunctional brain areas, such as the anterior cingulate cortex (ACC) and thalamus [48].

Another drawback is the lack of follow-up data after CSF shunting, and there is no doubt that this information is desired. To fill this gap, longitudinal information is being gathered to confirm the long-term improvement of the aforementioned cognitive aspects and their consistency over time.

\section{Acknowledgements}

Not applicable.

\section{Authors' contributions}

PAK: Design, conceptualization of the study, analysis or interpretation of the data, and revising the manuscript for intellectual content. RKS: Conceptualization of the study. MKP: Analysis and interpretation of the data. RR: Revising the manuscript for intellectual content. HAT: Revising the manuscript for intellectual content. Nothing to disclose. All authors report no disclosures. This paper has not been submitted elsewhere. All authors read and approved the final manuscript.

\section{Funding}

This research was done without any external funding.

\section{Availability of data and materials}

Not applicable.

\section{Declarations}

\section{Ethics approval and consent to participate}

The protocol was approved by the Ethics Committee of the Neurological Institute of Curitiba in Humans Research for having followed the principles of the Helsinki Convention and its later amendments, as well as Brazilian guidelines disposed by resolution No. 466/2012, all individuals were adequately consented. The committee's reference number is CAAE: 26239614.0.0000.5227.

\section{Consent for publication}

Not applicable.

\section{Competing interests}

The authors declare that they have no competing interests.

\section{Author details}

${ }^{1}$ Neurological Institute of Curitiba (INC), Curitiba, Street Jeremias Maciel Perretto, 300, Curitiba, Paraná 81210-310, Brazil. ${ }^{2}$ Headache Division and Pain Residence, Neurology Division, Hospital Clinics, Federal University of Paraná, Curitiba, Brazil. ${ }^{3}$ Neurology Service, Internal Medicine Department, Hospital Clinics, Federal University of Paraná, Curitiba, Paraná, Brazil.

Received: 31 January 2021 Accepted: 20 April 2021

Published online: 06 May 2021

\section{References}

1. Adams RD, Fisher CM, Hakim S, Ojemann RG, Sweet WH. Symptomatic occult hydrocephalus with normal cerebrospinal-fluid pressure. N Engl J Med. 1965;273:117-26.

2. Anderson M. Normal pressure hydrocephalus. Br Med J (Clin Res Ed). 1986;293(6551):837-8

3. Relkin N, Klinge P, Bergsneider M, Black PM. Diagnosing idiopathic normal-pressure hydrocephalus. Neurosurgery. 2005;57(Suppl 3):4-16.
4. Wikkelsö C, Andersson H, Blomstrand C, Lindqvist G, Svendsen P. Predictive value of the cerebrospinal fluid tap-test. Acta Neurol Scand. 1986;73:566-73.

5. Marmarou A, Bergsneider M, Klinge P, Relkin N, Black PM. The value of supplemental prognostic tests for the preoperative assessment of idiopathic normal-pressure hydrocephalus. Neurosurgery. 2005;57(Suppl 3):S17-28.

6. Ishikawa M, Hashimoto M, Mori E, Kuwana N, Kazui H. The value of the cerebrospinal fluid tap test for predicting shunt effectiveness in idiopathic normal pressure hydrocephalus. Fluids Barriers CNS. 2012;9(1):1.

7. Haan J, Thomeer RT. Predictive value of temporary external lumbar drainage in normal pressure hydrocephalus. Neurosurgery. 1988;22(2):388-91.

8. Walchenbach R, Geiger E, Thommer R, Vanneste J. The value of temporary external lumbar CSF drainage in predicting the outcome of shunting on normal pressure hydrocephalus. J Neurol Neurosurg Psychiatry. 2002;72(4):503-6.

9. Virhammar J, Cesarini KG, Laurell K. The CSF tap test in normal pressure hydrocephalus: evaluation time, reliability and the influence of pain. Eur J Neurol. 2012;19(2):271-6.

10. Squire $L R$, Wixted JT. The cognitive neuroscience of human memory since H.M. Annu Rev Neurosci. 2011;34:259-88.

11. Queenan BN, Ryan TJ, Gazzaniga MS, Gallistel CR. On the research of time past: the hunt for the substrate of memory. Ann NY Acad Sci. 2017;1396(1):108-25.

12. Muller GE, Schuman NF. Experimentelle beiträge zur untersuchung des gedächtmisses. Zeitschrift Für Psychologie Am J Psychol. 1984;6(2):299-301.

13. Pergher GK, Stein LM. Compreendendo o esquecimento: teorias clássicas e seus fundamentos experimentais. Psicol USP. 2003;14(1):129-55.

14. Wixted JT. The psychology and neuroscience of forgetting. Annu Rev Psychol. 2004;55:235-69.

15. Martins RT, Toson B, Souza RKM, Kowacs PA. Post-dural puncture headache incidence after cerebrospinal fluid aspiration. A prospective observational study. Arq Neuropsiquiatr. 2020;78(4):187-92.

16. Souza RKM, Rocha SFB, Martins RT, Kowcas PA, Ramina R. Gait in normal pressure hydrocephalus: characteristics and effects of the CSF tap test. Arq Neuropsiquiatr. 2018;76(5):324-31.

17. Benejam, B. Poca MA, Junqué C, Solana E, Sahuquillo J. Cognitive impairment in normal pressure hydrocephalus (NPH): a proposal for clinical evaluation protocol. Neurocirugia (Astur). 2008;19(4):309-21.

18. Kiefer $\mathrm{M}, \mathrm{Unterberg} A$. The differential diagnosis and treatment of normalpressure hydrocephalus. Dtsch Arztebl Int. 2012;109(1-2):15-25.

19. Bugalho P, Alves L, Miguel R, Ribeiro O. Profile of cognitive dysfunction and relation with gait disturbance in Normal Pressure Hydrocephalus. Clin Neurol Neurosurg. 2014;118:83-8.

20. Kazui H, Mori E, Ohkawa S, et al. Predictors of the disappearance of triad symptoms in patients with idiopathic normal pressure hydrocephalus after shunt surgery. J Neurol Sci. 2013;328(1-2):64-9.

21. Katzen H, Ravdin LD, Assuras S, et al. Postshunt cognitive and functional improvement in idiopathic normal pressure hydrocephalus. Neurosurgery. 2011;68(2):416-9.

22. Gleichgerrcht E, Cervio A, Salvat J, Loffredo AR, Vita L, Roca M, Torralva T, Manes F. Executive function improvement in normal pressure hydrocephalus following shunt surgery. Behav Neurol. 2009;21(3):181-5.

23. Saito M, Nishio Y, Kanno S, Uchiyama M, et al. Cognitive profile of idiopathic normal pressure hydrocephalus. Dement Geriatr Cogn Dis Extra. 2011;1(1):202-11.

24. Tullberg M, Hellström P, Piechnik SK, Starmark JE, Wikkelsö C. Impaired wakefulness is associated with reduced anterior cingulate CBF in patients with normal pressure hydrocephalus. Acta Neurol Scand. 2004;110(5):322-30.

25. Jones BE. Arousal systems. Front Biosci. 2003;1(8):438-51.

26. Brandelero V, Toni PM. Study of validity of Stroop color and word test for inhibitory control. Psicol Argum. 2015;33(80):282-97.

27. Isik AT, Kaya D, Ates Bulut E, Dokuzlar O, Soysal P. The outcomes of serial cerebrospinal fluid removal in elderly patients with idiopathic normal pressure hydrocephalus. Clin Interv Aging. 2019;14:2063-9.

28. Bradley WG, Whittemore AR, Watanabe AS, Davis SJ, Teresi LM, Homyak M. Association of deep white matter infarction with chronic communicating 
hydrocephalus: implications regarding the possible origin of normalpressure hydrocephalus. AJNR Am J Neuroradiol. 1991;12(1):31-9.

29. Tullberg M, Hultin L, Ekholm S, Månsson JE, Fredman P, Wikkelsø C. White matter changes in normal pressure hydrocephalus and Binswanger disease: specificity, predictive value and correlations to axonal degeneration and demyelination. Acta Neurol Scand. 2002;105(6):417-26.

30. Takeuchi T, Goto H, Izaki K, Tamura S, et al. Pathophysiology of cerebral circulatory disorders in idiopathic normal pressure hydrocephalus. Neurol Med Chir (Tokyo). 2007;47(7):299-306.

31. Bateman GA. The pathophysiology of idiopathic normal pressure hydrocephalus: cerebral ischemia or altered venous hemodynamics? AJNR Am J Neuroradiol. 2008;29(1):198-203.

32. Chen YF, Wang YH, Hsiao JK, et al. Normal pressure hydrocephalus: cerebral hemodynamic, metabolism measurement, discharge score, and long-term outcome. Surg Neurol. 2008;70(Suppl 1):69-77.

33. Spreen O, Strauss E. A compendium of neuropsychological tests: Administration, norms and commentary. New York: Oxford University. 1998;2:1240.

34. Kahlon B, Sundbärg G, Rehncrona S. Comparison between the lumbar infusion and CSF tap tests to predict outcome after shunt surgery in suspected normal pressure hydrocephalus. J Neurol Neurosurg Psychiatry. 2002;73(6):721-6

35. Schmidt H, Elster J, Eckert I, Wiefek J, et al. Cognitive functions after spinal tap in patients with normal pressure hydrocephalus. J Neurol. 2014;261(12):2344-50

36. Yamada S, Ishikawa M, Miyajima M, et al. Disease duration: the key to accurate CSF tap test in iNPH. Acta Neurol Scand. 2017;135(2):189-96.

37. Damasceno BP, Carelli EF, Honorato DC, Facure JJ. The predictive value of cerebrospinal fluid tap-test in normal pressure hydrocephalus. Arq Neuropsiquiatr. 1997;55(2):179-85.

38. Wikkels $\varnothing$ C, Hellström P, Klinge PM, Tans JT, European iNPH Multicentre Study Group. The European iNPH Multicentre Study on the predictive values of resistance to CSF outflow and the CSF Tap Test in patients with idiopathic normal pressure hydrocephalus. J Neurol Neurosurg Psychiatry. 2013;84(5):562-8

39. Williams MA, Malm J. Diagnosis and treatment of idiopathic normal pressure hydrocephalus. Continuum (Minneap Minn). 2016;22(2):579-99.
40. Solana E, Poca MA, Sahuquillo J, Benejam B, Junqué C, Dronavalli M. Cognitive and motor improvement after retesting in normal-pressure hydrocephalus: a real change or merely a learning effect? J Neurosurg. 2010;112(2):399-409.

41. Jutten RJ, Grandoit E, Foldi NS, Sikkes SAM, Jones RN, Choi SE, Lamar ML, Louden DKN, Rich J, Tommet D, Crane PK, Rabin LA. Lower practice effects as a marker of cognitive performance and dementia risk: A literature review. Alzheimers Dement (Amst). 2020;12(1):2055.

42. Cooper DBP, Lacritz LH, Weiner MF, Rosenberg RN, Cullum CM. Category fluency in mild cognitive impairment: reduced effect of practice in testretest conditions. Alzheimer Dis Assoc Disord. 2004;18(3):120-2.

43. Darby D, Maruff P, Collie A, McStephen M. Mild cognitive impairment can be detected by multiple assessments in a single day. Neurology. 2002;59(7):1042-6

44. Duff $K$, Beglinger $L$, Van Der Heiden $S$, et al. Short-term practice effects in amnestic mild cognitive impairment: implications for diagnosis and treatment. Int Psychogeriatr. 2008;20(5):986-99.

45. Duff K, Chelune G, Dennett K. Within-session practice effects in patients referred for suspected dementia. Dement Geriatr Cogn Disord. 2012;33(4):245-9.

46. Kito Y, Kazui H, Kubo Y, et al. Neuropsychiatric symptoms in patients with idiopathic normal pressure hydrocephalus. Behav Neurol. 2009;21(3):165-74.

47. Lindqvist $G$, Malmgren $H$. Organic mental disorders as hypothetical pathogenetic processes. Acta Psychiatr Scand Suppl. 1993;373:5-17.

48. Klinge PM, Brooks DJ, Samii A, et al. Correlates of local cerebral blood flow (CBF) in normal pressure hydrocephalus patients before and after shunting-A retrospective analysis of [(15)O]H(2)O PET-CBF studies in 65 patients. Clin Neurol Neurosurg. 2008;110(4):369-77.

\section{Publisher's Note}

Springer Nature remains neutral with regard to jurisdictional claims in published maps and institutional affiliations.

Ready to submit your research? Choose BMC and benefit from:

- fast, convenient online submission

- thorough peer review by experienced researchers in your field

- rapid publication on acceptance

- support for research data, including large and complex data types

- gold Open Access which fosters wider collaboration and increased citations

- maximum visibility for your research: over $100 \mathrm{M}$ website views per year

At BMC, research is always in progress.

Learn more biomedcentral.com/submissions 